\title{
Discussion on Ankang Shadow Play Art
}

\author{
Hongming $\mathrm{Xu}$
}

\author{
Department of Art, Ankang University, Ankang, 725000, China
}

\section{Keywords: Shadow play, Ankang shadow play, Shaannan Daoqing}

\begin{abstract}
Shadow play is an artistic expression with a long history in China and its origin and development have experienced a long time. Ankang Shadow Play is also called "Taiping play", "galanty show" and "shadow puppet" and its performance and size reduce with the decrease of performance needs. In addition to the above-mentioned basic structure and form, the composition and performance method of Ankang shadow play groups have different features and representatives in different counties. With Hanchu opera as the singing form, the unique performance technique of Hanyin Shadow Play is an independent school in China's shadow play troupes, which is typical and representative. There are many shadow play clubs in North District of Xunyang County, with frequent activities, represented by Fengshu Club and Xigouhe Club, who are famous in the local place and attach great importance to the plot expression and characterization as well as the combination of drama content and performance form. It is hoped that the emphasis on culture and art can completely preserve and save the shadow play and its related artistic form and language in the future development even if its original position cannot be regained.

Shadow play is an artistic expression with a long history in China and its origin and development have experienced a long time. At the same time, in the history, the shadow play art has formed its unique features in terms of craftsmanship, performance technique, recording mode and inheritance concept. The shadow play is a comprehensive art and has formed a set of unique artistic language and dissemination system from the production of props and background instruction and preparation of performance stage and site to the use of hand skills and special shadow props and to the design of aria, characters' lines and dialogue. Meanwhile, the different regional development leads to different artistic features. The shadow play art has different features in different regions. Shaanxi is an important province for the development of shadow play and northern Shaanxi, central Shaanxi plain and southern Shaanxi have shadow play groups and repertories with different characteristics. As an important central city of southern Shaanxi, Ankang's shadow play art has a long history and is popular in Hanbin District, Xunyang, Pingli, Ziyang, Hanyin, Shiquan and other county. There are more than 1200 traditional repertories, including a large number of historical stores and folk stories.
\end{abstract}

Ankang Shadow Play is also called "Taiping play", "galanty show" and "shadow puppet" and its performance and size reduce with the decrease of performance needs. The performance time is divided into fixed type and temporary type. For the fixed one, the local government or government units, with county, town and village as the unit, invites the nearby shadow play troupe to perform at the public place of town and village at festivals and at New Year. For example, Wuli Town invites local "Xinxing Troupe" or shadow play troupe of Hanyin troupe to perform at Qinglong Temple behind the village at the Spring Dragon Festival, the Double Ninth Festival, lunar January, Guanyu Festival and other festivals. With a long time and large size, such performance often lasts for 2-3 days and at the same time there are other forms of traditional performance. For the temporary one, individuals with family and village as the unit invites the theatrical troupe to perform at home for wedding and funeral.

The shadow play actors are similar to farmers at ordinary times and gathered by the leader or Lanmen in case of any performance, with simple and convenient performance forms and the props and tools of shadow play are often put in 1 or 2 wooden cases, with convenient carrying and transportation, so the local people also called the shadow play as "a shoulder pole play". The performance place varies with the situation of village or performance site, either large or small. However, the large site is only suitable for performance after dusk and the small central room and 
hall are suitable for performance at daytime after turning off the lights and closing the windows. At present, with Ankang as the center, the one district and nine counties have many shadow play groups, mainly called "XX troupe". There are 6-10 actors in the shadow play, among which there is only one actor that operates the shadow play figures, called "Lanmen". This actor is the core and also the main actor of the troupe and responsible for the performance, aria, dialogue and lines of shadow play in the performance, so also called "Xianshou". In the process of performance, there is few opportunity that he needs the help of "Lanmen disciple". Other actors are mainly responsible for the operation of different musical instruments and collective harmony. Taking "Xinxing Troupe" of Jianmin Town as an example, its actors and their works are as follows:

1. Lanmen - Yang Senlian, main actor, responsible for all shadow play and aria, also called "Xianshou"

2. Pixian - Tang Zhanggen, main member of instrument performance

3. Banhu - Chen Guangzhong, main member of instrument performance

4. Drum - Li Chengrong, main member of instrument performance

5. Small gong - Zhou Xingbao, main member of instrument performance

6. Flute - Zhou Xingbao, main member of instrument performance

7. Lanmen disciple - Liu Huazhong

At the same time, 2-3 reserve actors may conduct auxiliary operation according to the needs of repertory. During performance, the disciple of "Lanmen”, called "Lanmen disciple”, will also give a performance and at the same time, the disciple has to learn and practice the performance skills of his master, records the play content and learn the basic performance knowledge.

In addition to the above-mentioned basic structure and form, the composition and performance method of Ankang shadow play groups have different features and representatives in different counties. They will be introduced briefly as follows.

Shaannan shadow play is popular mainly in Hanyin County. With Hanchu opera as the singing form, the unique performance technique of Hanyin Shadow Play is an independent school in China's shadow play troupes, which is typical and representative. After about 100 years' historical changes, Hanyin Shadow Play skills have formed its unique charm in the fusion of immigration culture and north-south culture. Hanyin Shadow Play has been listed in the directory of Shaanxi Provincial non-material cultural heritage. The representative of Hanyin Shadow Play is Li Xingru, who is outstanding by virtue of its unique performance techniques and becomes a miracle in Ankang Shadow Play. In 2007, he was interviewed by CCTV7 and a feature news named Last Leader, lasting for more than 10 minutes, was recorded. In 2008, he was interviewed by China Folklore Network and a feature film named Exploring Folk Stunts, lasting for 20 minutes, was produced and broadcast on China Folklore Network. Li Xingru has standard performance form and forms a unique technique in many years' performance, such as rapid change of character face. For aria, Li Xingru has all-around singing technique and forms a unique singing form combined with Ankang traditional drama, Handiao-Er-Huang.

There are many shadow play clubs in North District of Xunyang County, with frequent activities, represented by Fengshu Club and Xigouhe Club, who are famous in the local place and attach great importance to the plot expression and characterization as well as the combination of drama content and performance form. With Shaannan Daoqing as the main singing tune, Xunyang Shadow Play also has Ankang traditional "Handiao-Er-Huang”. For Xunyang Shadow Play, the Daoqing tune is mainly banqiang style and the lyrics mainly have seven or ten words. As needed, some folk ditties and the names of the tunes of other types of drama are adopted sometimes. The characteristic tune in Daoqing is "Huangma", for which when the Lanmen or main singer sings a piece of lyrics, other musical instruments or prop players sing a chant together, corresponding to the former lyrics and creating a busy atmosphere. In Xunyang Shadow Play, there is a tune called "Babujing", which is popular in Fengshuping, junction of Xunyang and Zhen'an. The rhythm of "Babujing" is similar to talking and singing of percussion music.

In addition to the shadow play clubs in each county of Ankang, there are some clubs in the 
villages and towns that perform shadow play. There is "Xinxing Troupe" in Jianmin Town, Ankang City. The lanmen of "Xinxing Troupe" is Yang Senlian, who was born in Jianmin Town, Hanbin District in 1928 and four generations of his family have engaged in shadow play art. He went to school for 2 years in his childhood, then learned knowledge and skills from his father, Yang Tianchang, at the age of 10, with script as the textbook and went up on the stage at the age of 12 as Lanmen. He was honest and kind and paid attention to unify other actors. With all-around technique, he was popular in the local place and had more than 300 plays and "shelf" plays. "Xinxing Troupe" was established by Yang Senlian after the new century, in which yang Senlian is the Lanmen and Tang Zhanggen is responsible for producing shadow play props and also the player of Pixian. In Hanbin District, center of Ankang City, there is no fixed shadow play group, but there are some enthusiasts that collect shadow play props and performance props and have collected a considerable number of Ankang shadow play props.

With the development of the times, shadow play gradually fades out from the life of ordinary people. The reduce of performance groups, times and income do not only make the development of Shaannan shadow play art, represented by Ankang, stagnate and gradually miss, but also make the collection and preservation of shadow play art data hard. It is hoped that the emphasis on culture and art can completely preserve and save the shadow play and its related artistic form and language in the future development even if its original position cannot be regained.

\section{Acknowledgments}

This paper is the research result of "Research on Ankang Shadow Play Art" of Shaanxi Provincial Education Department, number: 12JK0330.

\section{References}

[1] Pan Jialai. Chinese Traditional Shadow Play[M]. Beijing: People's Fine Arts Publishing House, 2004.

[2] Wei Liqun. History of Chinese Shadow Play Art[M]. Beijing: Cultural Relics Press, 2007.

[3] Yang Fei. Shaanxi Shadow Play[M]. Xi’an: Sanqin Press, 2003.

[4] Shen Wenxiang, Lu Ping. Appreciation of Shaanxi Shadow Play[M]. Beijing: Wenhui Press, 2007. 